\title{
Pathology-based approach to epilepsy surgery
}

\author{
Ingmar Blumcke • Giorgio Lo Russo • Imad Najm • \\ Andre Palmini
}

Published online: 31 May 2014

(C) Springer-Verlag Berlin Heidelberg 2014

Despite the introduction of scores of antiepileptic medications over the last two decades, a sizable number of patients (almost one-third) with epilepsy continue to have seizures. In fact, the growing realization that pharmaco-resistant epilepsy carries both a significant mortality risk and lingering psycho-social stigmata highlights the challenge to provide effective medical treatment for a significant percentage of patients. In this group of patients with pharmaco-resistant focal epilepsy, resective surgery has become the treatment of choice.

Long-term follow-up studies on the success and complications of epilepsy surgery have been published by various centers of excellence. These studies showed the efficacy of resective surgery in seizure control and established new outcome "benchmarks". These positive developments and the expertise gained by the epilepsy community in managing difficult to medically treat epilepsy patients, led to a significant increase in the number of epilepsy programs around the world and heightened awareness of referring physicians and patients about the benefit of epilepsy surgery. Furthermore, there have been advances in neurophysiological and neuroimaging evaluation protocols, and

\footnotetext{
I. Blumcke $(\bowtie)$

University of Erlangen School of Medicine, Erlangen, Germany

e-mail: bluemcke@uk-erlangen.de
}

G. L. Russo

Epilepsy Surgery Centre C. Munari, Milan, Italy

I. Najm

Epilepsy Center, Neurological Institute, Cleveland Clinic, Cleveland, $\mathrm{OH}, \mathrm{USA}$

A. Palmini

Porto Alegre Epilepsy Surgery Program, Pontificia Universidade Católica do Rio Grande do Sul, Porto Alegre, Brazil surgical candidacy has broadened as a consequence. However, even twenty-first century technology still falls vexingly short of solving the problem for many patients.

The role of specialists is to seek solutions, and explore paradigm shifts that often proves useful to advance knowledge and decision-making. Historically, the epilepsy surgery 'jargon' has been one of dividing the brain into anatomical compartments and exploring the corresponding types of epilepsies based on the localization of the epilepsy onset (e.g., temporal, frontal, parieto-occipital lobe epilepsies). Terms such as temporal lobe or frontal lobe epilepsies became entrenched in the literature and in the mind of those evaluating and operating on patients. Outcomes, however, have been widely diverse and, to some extent, continue to be suboptimal.

Focal epilepsy as a clinical phenotype could be considered as the final common pathway of various and diverse etiologies that include focal lesions (tumors, scars, vascular malformations, or cortical dysplasia), multilobar lesions (cortical dysplasia, tuberous sclerosis, destructive lesions), hemispheric (hemimegalencephaly, Rasmussen's encephalitis) or bihemispheric (cortical dysplasia, tuberous sclerosis). An increased knowledge of the natural history of epilepsies due to different lesions, of the clinical/ electrophysiological/imaging presentations and treatment outcomes of these epilepsies suggest distinct characteristics that go beyond the anatomical location of epilepsy phenotype.

In this topic-related cluster of review articles, we claim that development of epilepsy (epileptogenesis) and expression of epilepsy (epileptogenicity) largely depend on causative abnormality(ies) in patients suffering from pharmaco-resistant focal epilepsies: previous studies addressing presurgical evaluation and postsurgical outcome highlighted important roles for the interactions between the 
epilepsy pathology and both adjacent and distant brain regions. Some pathologies more easily recruit additional regions in newly formed epileptogenic networks; whereas, other lesions lead to localized, contiguous epileptogenesis. In short, specific types of epileptic lesions pose specific challenges for the localization, definition of extent, functional/electrophysiological mapping and resection of the relevant epileptic brain and lesional "proepileptic" tissue. These characteristics, in addition to the long-term postsurgical outcomes achieved in these patients, largely depend on the causative lesion rather than its location in the brain.

Therefore, we believe the time is ripe to switch the epileptological perspective from an anatomy (location)-centric towards a pathology (lesion/etiology)-centric approach. This proposed shift would lead to a better understanding of the epileptic/functional behavior of the lesion and predict its natural history (and thus treatment outcomes). From a presurgical assessment standpoint, the localization of epileptic focus (in particular, in MRI-negative cases or in patients with multiple or diffuse lesions) requires a "global", "trans-lobar" approach based on three-dimensional anatomo-functional brain networks. This is done through analysis of the sequence of clinical events observed in various types of seizures (usually independent from the causative pathology). Any change in the clinical expression over time of the seizures, while pointing to the non static nature of epilepsy, would suggest a change in the epileptic three dimensional network.

The 21st International Epilepsy Surgery Symposium, co-organized by the epilepsy groups at the Cleveland Clinic, the Niguarda Hospital and Besta Institute in Milan and a consortium of centers in Brazil aimed to explore the pathology-based paradigm. Sessions and discussions were organized according to putative causative epilepsy pathology with debates on the possible mechanisms of epilepsy generation, clinical presentation and natural history, presurgical evaluation and surgical treatment approach and options. Therefore, the various discussions by a large group of international experts in the field revolved around the cause of epilepsy generation in the various brain locations, and not the other way around.

The first contribution by Najm and colleagues reflects the increasing awareness and interest in Focal Cortical Dysplasias as underlying cause of drug-resistant focal epilepsies in children and young adults, presenting either with or without specific imaging abnormalities. Emerging data on electro-functional and imaging correlations in various subtypes of FCDs and their implications on both surgical management and outcomes after resection is thoroughly reviewed and discussed. However, the broad spectrum of histopathological lesion patterns as well as the various clinical phenotypes continue to present some challenges in the classification of FCD subtypes. These persisting shortcomings are discussed in this article using the first international consensus classification system of FCDs, as proposed by the International League against Epilepsy (ILAE) in 2011.

Hippocampal Sclerosis (HS) represents one of the most frequent histopathological abnormalities encountered worldwide in published series of patients submitted to epilepsy surgery. In 2013, ILAE proposed a first international consensus classification of clinico-pathological HS subtypes, which is reviewed by Cendes and colleagues in the second article of this cluster. The authors suggest that a more comprehensive knowledge about distinct clinico-pathological subtypes of HS will switch our attention towards a differentiated view of potential etiologies, although current knowledge about causes and consequences as well as targeted clinical management remains largely unknown. The ILAE classification of HS will also help to foster new research avenues towards use of more refined animal models and human brain tissues as most promising strategies to improve our understanding of HS and its accompanied long-term consequences, such as cognitive impairment due to structural lesions in the hippocampus.

Brain tumors represent the third most frequent lesion pattern in various series of patients with pharmaco-resistant focal epilepsies. Such patients have their seizure onset usually in childhood or adolescence, and medical management requires strategies different from those established for adult neurooncology patients. The third article discusses a critical issue in the approach of patients with brain tumors and epilepsy that requires an understanding of the epileptogenicity created by the tumor. Chronic in situ epileptogenicity caused by tumors spans beyond the proper histological tumor margin. This is highlighted in reports on lack of seizure control after complete removal of the tumor without resection of additional epileptic tissue. Therefore, the management of these patients requires an "epilepsy surgery" rather than a "tumor surgery" approach; whereas, the identification, localization and mapping of the epileptogenic areas and their removal (in addition to tumor resection) are the two fundamental components of the "epilepsy surgery" in tumor-associated epilepsies. Another challenge consists in the neuropathological diagnosis of these earlyonset and long-term epilepsy associated tumors (LEAT) as their often bizarre and variable histopathology can be easily overinterpreted. Blümcke and colleagues will propose a new approach for the neuropathological terminology of LEAT, taking into account more specific histopathological biomarkers as well as recent molecular-diagnostic studies. As the authors agree that the time may be too early for a new WHO classification of LEAT, there is a definite need to start a comprehensive discussion between the international community of neuropathologists and epileptologists for a better prediction of each tumor's biological nature and 
epileptogenic potential through direct histopathologicalelectro-clinical correlations (for details look at the new A-B-C LEAT terminology).

Jehi and colleagues will conclude this cluster by a comprehensive review on vascular malformations in the setting of chronic epilepsies. Cavernomas are important causes of pharmaco-resistant focal epilepsy. New management guidelines of these lesions have been recently proposed by an ad hoc Task Force of the ILAE. As these guidelines will certainly need validation by randomized, prospective, clinical trials, the comprehensive work-up of large patient series obtained from two experienced centers at Cleveland Clinic and from the Porto Alegre Epilepsy Surgery Program in
Brazil will be most helpful for our understanding of persisting challenges for treatment of epilepsy when related to vascular malformations.

In this "cluster", we present an abridged view of main topics of interest as well as discussions raised at the $21 \mathrm{st}$ International Epilepsy Symposium for the purpose of improving the outcome of epilepsy surgery. We believe the cluster will be of interest for the entire community of neuropathologists and epileptologists, as well as related disciplines with clinical and research perspectives to recast thinking about refractory focal epilepsies and the way to make decisions toward localizing and mapping the relevant epileptogenic brain tissue and to improve surgical treatment. 